\title{
Estudo de vazões máximas de projeto usando uma distribuição de Gumbel
}

\author{
Study of maximum flows of project using a Gumbel distribution \\ Estudio de los flujos máximos de proyecto utilizando una distribución de Gumbel
}

Recebido: 01/09/2021 | Revisado: 08/09/2021 | Aceito: 14/09/2021 | Publicado: 15/09/2021

\author{
Maria Rianne Pereira Nobre \\ ORCID: https://orcid.org/0000-0003-1511-7407 \\ Centro Universitário INTA, Brasil \\ E-mail: mariariannenobre@ hotmail.com \\ Maurício de Sousa Pereira \\ ORCID: https://orcid.org/0000-0002-5485-1674 \\ Centro Universitário INTA, Brasil \\ E-mail: mauricio.pereira@uninta.edu.br \\ Rogério Campos \\ ORCID: https://orcid.org/0000-0003-2762-5484 \\ Centro Universitário INTA, Brasil \\ E-mail: rogerio.campos@uninta.edu.br
}

\begin{abstract}
Resumo
O cálculo de cheias máximas de projeto é fundamental para o projeto e desenvolvimento de construções hidráulicas. A previsão de enchentes, por exemplo, pode ser estimada, aplicando o cálculo de uma enchente de projeto por extrapolação dos dados históricos para condições extremas. Assim, para evitar riscos de colapso em construções hidráulicas, é fundamental o uso de distribuições estatísticas especializadas para realizar cálculos de vazões de projeto de vertedouros. A distribuição de Gumbel é uma das funções de distribuição de probabilidade mais amplamente utilizadas para valores extremos em estudos hidrológicos, nos quais os parâmetros considerados na análise são a vazão e período de retorno. Neste trabalho de pesquisa, uma distribuição de Gumbel foi utilizada para analisar os dados das vazões máximas de uma série histórica da estação Tabajara, do rio Ji-Paraná, localizado no município de Machadinho d'Oeste - RO. Os dados estudados foram coletados entre os anos de 1978 e 2015.
\end{abstract}

Palavras-chave: Distribuição de Gumbel; Valores extremos; Vazões máximas; Período de retorno.

\begin{abstract}
The calculation of project maximum floods is fundamental for the design and development of hydraulic constructions. Flood forecasting can be estimated, for example, by applying the calculation of a project flood by extrapolating historical data to critical conditions. Thus, to avoid risks of collapse in hydraulic constructions, it is essential to use specialized statistical distributions to perform the calculations of flows of spillways project. The Gumbel distribution is one of the most widely used probability distribution functions for extreme values in hydrological studies, in which the parameters considered in the analysis are the flow and return period. The Gumbel distribution is one of the most widely used probability distribution functions for extreme values in hydrological studies, in which the parameters considered in the analysis are the flow and return period. In this research work, a Gumbel distribution was used to analyze the data of the maximum flows of a historical series of the Tabajara station, of the Ji-Parana river, located in the city of Machadinho d'Oeste - RO. The data studied were collected between 1978 and 2015.
\end{abstract}

Keywords: Gumbel distribution; Extreme values; Maximum flows; Return period.

\section{Resumen}

El cálculo de las crecidas máximas de diseño es fundamental para el diseño y desarrollo de construcciones hidráulicas. El pronóstico de inundaciones, por ejemplo, se puede estimar aplicando el cálculo de una inundación del proyecto extrapolando datos históricos a condiciones extremas. Por lo tanto, para evitar riesgos de colapso en las construcciones hidráulicas, es esencial utilizar distribuciones estadísticas especializadas para realizar los cálculos de flujo del diseño del aliviadero. La distribución de Gumbel es una de las funciones de distribución de probabilidad más utilizadas para valores extremos en estudios hidrológicos, en los que los parámetros considerados en el análisis son el caudal y el período de retorno. En este trabajo de investigación, se utilizó una distribución de Gumbel para analizar los datos de caudal máximo de una serie histórica de la estación Tabajara, en el río Ji-Paraná, ubicada en el municipio de Machadinho d'Oeste - RO. Los datos estudiados fueron recolectados entre 1978 y 2015.

Palabras clave: Distribución de Gumbel; Valores extremos; Caudales máximos; Periodo de devolución. 


\section{Introdução}

Geralmente, em projetos de recursos hídricos, o planejamento e design do empreendimento visa determinar a magnitude e a frequência das inundações que poderão ocorrer nas áreas do projeto. Assim, compreender a variabilidade dos fenômenos hidrológicos se torna fundamental para prever catástrofes naturais e auxiliar nos estudos de eventos climáticos extremos. Nesse contexto, a abordagem probabilística para valores extremos de processos hidrológicos representa um grande progresso na ciência e engenharia hidrológica, uma vez que possibilita mapear os riscos decorrentes desses potenciais eventos (Koutsoyiannis, 2004; Bhagat, 2017).

Estudos probabilísticos de variáveis hidrológicas como precipitações intensas e inundações constituem elementos importantes para apoiar o planejamento e gestão de recursos hídricos (Júnior et al., 2015; Marques et al., 2014; Neta et al, 2019). Devido à sua simplicidade e generalidade, as distribuições de valores extremos limitantes se tornam muito difundidas na hidrologia. Várias pesquisas têm investigado modelos de distribuição de probabilidade para valores extremos de variáveis climáticas. Os modelos baseados nas distribuições de Gumbel (Koutsoyiannis, 2004; Bhagat, 2017; Ganamala \& Kumar, 2017) e de valores extremos generalizados (Generalized Extreme Value, GEV) (Koutsoyiannis, 2004; Franco et al., 2014), têm sido utilizados com êxito para esse propósito.

O fato de a distribuição de Gumbel ser um dos modelos probabilísticos mais comuns usado na modelagem de extremos hidrológicos, especialmente de precipitações, pode ser interpretado como uma resposta positiva (Júnior et al., 2015). Além do mais, a estimativa de precipitações extremas é muito importante para o planejamento das estruturas hidráulicas, uma vez que as inundações projetadas são geralmente estimadas com base nos valores extremos das precipitações (Koutsoyiannis, 2004).

A avaliação de eventos extremos em bacias hidrográficas está associada a um estudo sistemático abrangendo as variáveis hidrológicas necessárias para o projeto de dimensionamento das obras hidráulicas, objetivando reduzir os riscos catástrofes e impactos ambientais potencializados pela obra. Uma das abordagens que pode ser realizada antes da elaboração de um projeto hidráulico de contenção é uma análise probabilística das vazões máximas suportadas pelo reservatório e das vazões previstas no projeto.

A previsão de vazões pode ser obtida realizando uma análise de vazões de projeto a partir da extrapolação de uma série histórica de dados em condições críticas. Além disso, as cheias de projeto são imprescindíveis para o dimensionamento de estruturas hidráulicas, e tem importância decisiva nos custos e na segurança dos projetos de engenharia. Por isso, projetos de engenharia e obras de controle são feitos com objetivo de evitar a inundação não planejada.

Neste trabalho de pesquisa foi realizado uma análise probabilística do cálculo de vazões de projeto utilizando uma distribuição de Gumbel para valores extremos, objetivando avaliar a frequência de distribuição de vazões máximas, estimar cheias de projeto e vazões de projeto de vertedouros. A equação de distribuição de Gumbel depende da adequação de uma função teórica de distribuição de probabilidade à uma série histórica de vazões em um dado local. A análise desta equação estabelece a relação entre vazões máximas e os tempos de retornos de ocorrência.

\section{Análise da Frequência de Cheias}

A previsão de eventos extremos, como as inundações, é de fundamental importância, para o planejamento e elaboração de projetos de estruturas hidráulicas. Estudos anteriores a elaboração de um projeto devem considerar as peculiaridades da região de implantação, a utilidade da construção e o desempenho da obra hidráulica. Elementos intrínsecos as bacias hidrográficas, por exemplo, estão intimamente ligadas aos processos de escoamento, infiltração, armazenamento da água e da precipitação pluviométrica (Soares et al., 2020). 
Existem diversos modelos que podem ser usados para determinar a frequência hidrológica das cheias, precipitações máximas e intensidade-duração-frequência de um evento. Em geral, os modelos probabilísticos dependem do uso de dados existentes para fazer previsões cenário futuro (Affonso et al., 2020; Filho et al., 2020; Ximenes et al., 2020).

A análise de frequência de cheias (AFC) é a estimativa de quantas vezes um evento especificado ocorrerá dentro de um determinado período. Antes que a estimativa possa ser feita, a análise dos dados das vazões dos rios é importante para se obter a distribuição da probabilidade de inundação. A distribuição de Gumbel (tipo I) é adequada para estimar eventos de tipo máximo AFC fornece a curva do modelo de probabilidade para os picos de cheias anuais que são registados pela observação do período e dados de cheias ocorridas em um determinado período. Este método é útil para prever o intervalo de recorrência desses eventos e, pode ajudar no planejamento e estratégia a serem adotadas para evitar ou reduzir os potenciais danos (Gamala \& Kumar, 2017; Kamal et al., 2017).

\section{Tempo de Retorno}

O estudo de eventos hidrológicos permite estimar, entre outras características, o tempo de recorrência de determinado evento como chuvas, cheias de rios e inundações. Essa análise se baseia a partir de bases históricas e cálculos probabilísticos, que é de fundamental importância, principalmente, quando se trata de eventos extremos (Jesus \& Nascimento, 2020).

A Lei de Segurança de Barragens estipula parâmetros para segurança relacionados aos impactos gerados e possibilidade de ruptura. Os danos são econômicos, sociais e ambientais, depende-se da área do risco (Comitê Brasileiro de Barragens, 2001). A Tabela 1 mostra a classificação da vazão de acordo com o risco potencial.

Tabela 1. Classificação da vazão de projeto de acordo com o risco.

\begin{tabular}{cccc}
\hline $\begin{array}{c}\text { Consequências da } \\
\text { ruptura }\end{array}$ & Perdas vitais & $\begin{array}{c}\text { Danos econômicos, } \\
\text { sociais e ambientais }\end{array}$ & $\begin{array}{c}\text { Cheia afluente de } \\
\text { projeto }\end{array}$ \\
\hline Muito Alta & Significativa & Excessivo & Cheia Máxima Provável \\
Alta & Alguma & Substancial & Cheia Excepcional \\
Baixa & Nenhuma & Moderado & $\mathrm{T}_{\mathrm{r}}=10000$ anos \\
Muito Baixa & Nenhuma & Nenhum & $* * *$ \\
\hline
\end{tabular}

Fonte: CBB (2001).

Para avaliar se uma determinada estrutura pode resistir a um evento no seu valor extremo é necessário realizar previsões e, isso pode ser feito a partir de cálculos probabilísticos considerando os principais parâmetros que determinam esse evento. Por exemplo, para organização de um sistema de alerta contra enchentes e para o planejamento das áreas de risco de um município, é importante que o período de retorno esteja relacionado aos níveis de observação, atenção e alerta uma maior segurança das áreas prejudicáveis.

Período de retorno (PR), também conhecido como intervalo de recorrência ou tempo de recorrência (TR), é o intervalo estimado (em anos) entre ocorrências de igual magnitude de um fenômeno natural, como chuvas, ventos intensos, granizo, entre outros (Gamala \& Kumar, 2017).

Quanto maior a obra, maior o risco e, consequentemente, maior deve ser o período de retorno. A Tabela 2 mostra o TR admitidos para algumas obras hidráulicas. 
Tabela 2. Período de retorno admitido para diferentes obras hidráulicas.

\begin{tabular}{lc}
\hline \multicolumn{1}{c}{ Obra Hidráulica } & Período de retorno (anos) \\
\hline Bueiros & 2 a 10 \\
Galerias de águas pluviais & 5 a 20 \\
Pequenas barragens de concreto para & \\
abastecimento de água & 50 a 100 \\
Canalização a céu aberto para trapezoidal & 50 \\
Canalização a céu aberto retangular & 100 \\
Travessias: pontes, bueiros e estruturas afins & 100 \\
Barragem de concreto & 500 \\
Extravasor de uma barragem de terra & 1000 \\
Vertedor de grandes barragens & 10.000 \\
\hline
\end{tabular}

Fonte: CBB (2001).

Para obter a expressão para o período de retorno, considera-se que a vazão de projeto tenha magnitude $\mathrm{x}_{\mathrm{p}}$, com intervalo de recorrência $T_{\mathrm{r}}$. A possibilidade de que a vazão seja igual ou superior, em algum ano, é expressa pela equação

$$
P\left\{X \geq x_{p}\right\}=1 / T_{r}
$$

Quando uma obra hidráulica for construída para uma vazão de projeto $\mathrm{x}_{\mathrm{p}}$, correspondente a um intervalo de recorrência $T_{\mathrm{r}}$ anos, para cada ano de atividade do sistema, a probabilidade da ocorrência de uma falha, a vazão ser superada é igual a $1 / \mathrm{T}_{\mathrm{r}}$.

Assim, a probabilidade da não ocorrência da falha em um algum ano será expressa pela equação

$$
P\left\{X \geq x_{p}\right\}=1-1 / T_{r}
$$

\section{Método de Gumbel}

Esta distribuição de valor extremo foi introduzida por Gumbel (1941) e é comumente conhecida como distribuição de Gumbel. Gumbel é uma das funções de distribuição de probabilidade mais amplamente utilizadas para valores extremos em estudos hidrológicos para previsão de precipitação máxima (Gumbel, 1941).

A distribuição de valores extremos do Tipo I recebeu as seguintes outras denominações: distribuição de Gumbel, Fisher-Tippet tipo I e dupla exponencial. No caso de valores máximos, a distribuição de Gumbel refere-se a forma assintótica limite para um conjunto de $\mathrm{N}$ variáveis aleatórias originais $\left\{\mathrm{X}_{1}, \mathrm{X}_{2}, \ldots, \mathrm{X}_{\mathrm{N}}\right\}$, independentes e igualmente distribuídas conforme um modelo $\mathrm{F}_{\mathrm{X}}(x)$, de cauda superior exponencial. A distribuição de Gumbel (máximos) é a distribuição extremal mais usada na análise de frequência de variáveis hidrológicas, com inúmeras aplicações na determinação de relações intensidade-duraçãofrequência de precipitações intensas e estudos de vazões de enchentes (Naghettini \& Pinto, 2007; Pereira \& Caldeira, 2018).

A função acumulada de probabilidades (FAP) da distribuição de Gumbel e dada pela relação

$$
F_{Y}(\mathrm{y})=\exp \left[-\exp \left(\frac{y-\beta}{\alpha}\right)\right] .
$$

na qual, $\alpha$ representa o parâmetro de escala e $\beta$ o parâmetro de posição. 
A função da densidade da distribuição de Gumbel é representada na equação

$$
f_{Y}(y)=\frac{1}{\alpha} \exp \left[-\frac{y-\beta}{\alpha}-\exp \left(\frac{y-\beta}{\alpha}\right)\right]
$$

$\mathrm{O}$ valor esperado, a variância e o coeficiente de assimetria de $\mathrm{X}$ são, respectivamente: $\mathrm{E}[\mathrm{Y}]=\beta+0,5772 \alpha, \operatorname{Var}[\mathrm{Y}]=$ $\sigma_{y}^{2}=\left(\frac{\pi^{2} \alpha^{2}}{6}\right)$ e $\gamma=1,1396$. Observe, portanto, que a distribuição Gumbel (máximos) possui um coeficiente de assimetria positivo e constante.

A função inversa da FAP de Gumbel, ou função de quantis, e expressa por:

$$
y(F)=\beta-\alpha \ln [-\ln (F)]
$$

ou

$$
y(F)=\beta-\alpha \ln \left[-\ln \left(1-\frac{1}{T_{r}}\right)\right]
$$

na qual, $\mathrm{T}_{\mathrm{r}}$ denota o período de retorno em anos e $\mathrm{F}$ representa a probabilidade anual de não superação.

\section{Metodologia}

Com o auxílio do software Excel foi elaborado uma planilha eletrônica, para o tratamento e análise dos dados estudados nesse trabalho de pesquisa em forma de um estudo de caso (Estrela, 2018). Para analisar o método da distribuição de Gumbel, utilizou-se uma série temporal das vazões da estação Tabajara, do rio Ji-Paraná ou Machado, localizado no município de Machadinho d'Oeste - RO (Latitude ( $\left.-08^{\circ} 56^{\prime} 00^{\prime \prime}\right)$, Longitude ( $-62^{\circ} 03^{\prime} 14^{\prime \prime}$ ).

Os dadas das vazões analisadas estão catalogados no banco de dados da Agência Nacional das Águas (ANA) no período compreendido entre 1978 e 2015.

Os dados das vazões e anos foram organizados na planilha de cálculo deste trabalho, seguindo a ordem cronológica. O número de eventos considerados foram 34, tendo início no ano de 1978 e término no ano de 2015. A vazão máxima da série estudada corresponde a $6200 \mathrm{~m}^{3} / \mathrm{s}$ no ano de 2013 .

\section{Resultados e Discussão}

\subsection{Vazões Máximas de Teste}

Inicialmente, foram realizados testes de vazões para tempos de retornos específicos. Nessa etapa foram calculadas as variáveis de teste e os parâmetros de distribuição teste. Os valores obtidos para as variáveis de teste, média, desvio padrão e variância, foram 12,50, 3,54 e 12,50, respectivamente. Enquanto, para os dos parâmetros de distribuição foram obtidos $\alpha=$ 2,76 e $\beta=10,91$. Para o período de retorno, foram utilizados 10, 50, 100, 200, 250, 500, 750, 1000, 2000, 4000, 6000, 8000 e 10.000 anos.

O cálculo das vazões foi realizado usando a função inversa da FAP de Gumbel (Equação 6) e os resultados obtidos estão representados na Tabela 3 e plotados na Figura 1. 
Tabela 3. Valores das vazões de teste máximas.

\begin{tabular}{cccc}
\hline Período de retorno (anos) & Vazões $\left(\boldsymbol{m}^{\mathbf{3}} \mathbf{s}\right)$ & Período de retorno $(\operatorname{anos})$ & Vazões $\left(\boldsymbol{m}^{\mathbf{3}} \mathbf{s}\right)$ \\
\hline 10 & 17,11 & 1000 & 29,95 \\
50 & 21,67 & 2000 & 31,86 \\
100 & 23,60 & 4000 & 33,77 \\
250 & 26,12 & 6000 & 34,89 \\
500 & 28,04 & 8000 & 35,68 \\
750 & 29,16 & 10000 & 36,30 \\
\hline
\end{tabular}

Fonte: Autores.

Figura 1. Distribuição de Gumbel para as vazões de teste máximas.

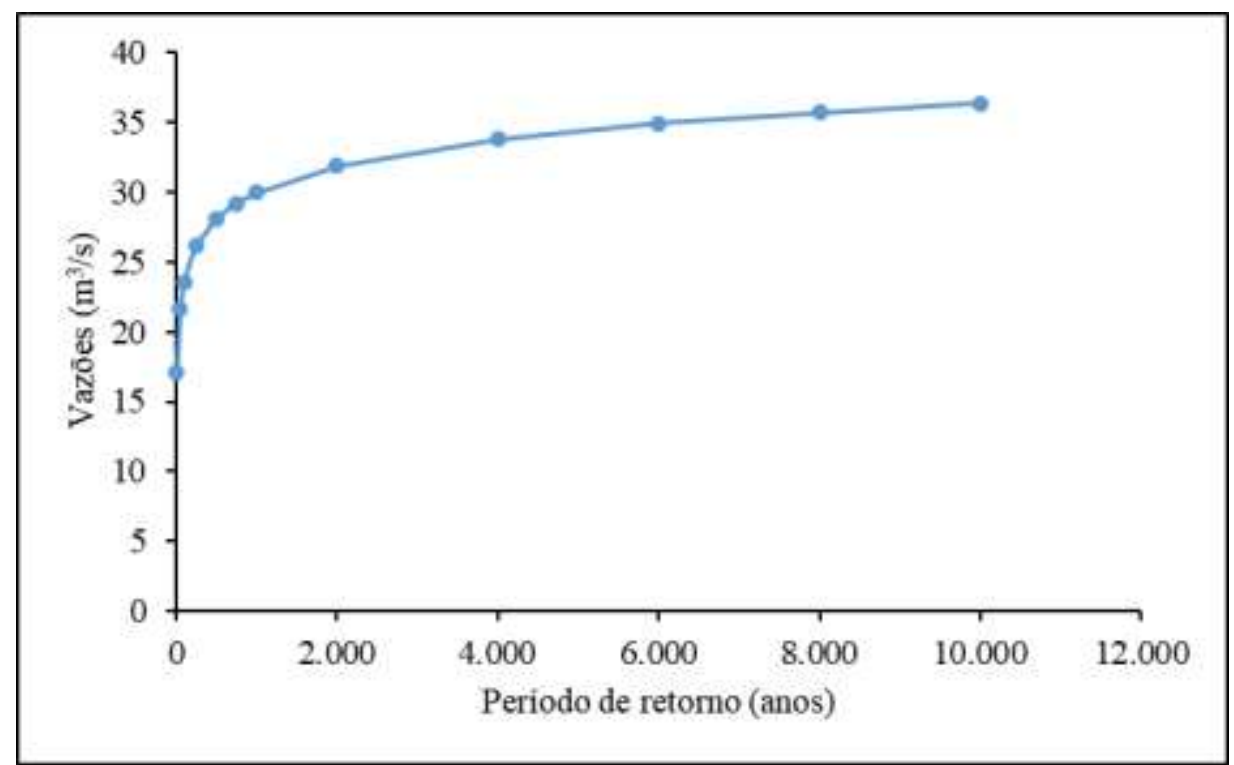

Fonte: Autores.

A partir do gráfico da Distribuição de Gumbel, pode-se observar que, quanto maior a chance de uma extrapolação do valor da vazão, maior é o período de retorno que deve ser levado em consideração para o cálculo em projetos hidráulicos, para uma não-ocorrência de falha. Com isso, tem-se o objetivo de se evitar catástrofes por uma extrapolação de condições críticas.

\subsection{Vazões de Projeto: Estudo de Caso}

Os valores das vazões para o referido estudo de caso estão de acordo com a Tabela 4. 
Tabela 4. Série temporal das vazões máximas da estação Tabajara.

\begin{tabular}{cccc}
\hline Ano & Vazões $\left(\boldsymbol{m}^{3} / \boldsymbol{s}\right)$ & Ano & Vazões $\left(\boldsymbol{m}^{3} / \boldsymbol{s}\right)$ \\
\hline 1978 & 4306,00 & 1999 & 3631,00 \\
1979 & 3469,00 & 2000 & 4946,00 \\
1980 & 3082,00 & 2001 & 3681,00 \\
1981 & 3766,00 & 2002 & 5055,00 \\
1982 & 3793,00 & 2003 & 4162,00 \\
1983 & 3046,00 & 2004 & 4569,00 \\
1984 & 3460,00 & 2005 & 4496,00 \\
1985 & 4549,00 & 2006 & 3855,00 \\
1986 & 3505,00 & 2007 & 4284,00 \\
1987 & 4126,00 & 2008 & 4284,00 \\
1989 & 5400,00 & 2009 & 5500,00 \\
1993 & 4549,00 & 2010 & 4001,00 \\
1994 & 3793,00 & 2011 & 4515,00 \\
1995 & 4915,00 & 2012 & 4558,00 \\
1996 & 5039,00 & 2013 & 6200,00 \\
1997 & 3703,00 & 2014 & 5373,00 \\
1998 & 3766,00 & 2015 & 4571,00
\end{tabular}

Fonte: Autores.

A média, o desvio padrão e a variância calculados a partir das vazões mostradas na Tabela 4 foram 4.316,79, 740,16 e $547.833,02$, respectivamente. Enquanto, para os dos parâmetros de distribuição foram obtidos $\alpha=577,12$ e $\beta=3.983,68$.

Realizado o cálculo dos parâmetros, foram calculados valores para 12 vazões máximas usando a distribuição de Gumbel. Os períodos de retornos mínimos e máximos adotados para o estudo foram 10 e 10000 anos, respectivamente. Os valores das vazões obtidas estão listados na Tabela 5. Além disso, os valores das vazões calculados foram, também, comparados com dados relatados na literatura (Mercadante, 2018).

Tabela 5. Comparação dos valores das vazões obtidos no estudo de caso x literatura.

\begin{tabular}{ccc}
\hline $\begin{array}{c}\text { Período de retorno } \\
\text { (em anos) }\end{array}$ & $\begin{array}{c}\text { Vazões }\left(\boldsymbol{m}^{3} / \mathbf{s}\right) \\
(\text { Estudo } \text { de } \text { caso })\end{array}$ & $\begin{array}{c}\text { Vazões }\left(\boldsymbol{m}^{\mathbf{3}} / \mathbf{s}\right) \\
\text { (Mercadante, 2018) }\end{array}$ \\
\hline 10 & $5.282,40$ & $5.280,00$ \\
50 & $6.235,55$ & $6.240,00$ \\
100 & $6.638,50$ & $6.640,00$ \\
500 & $7.169,05$ & --- \\
750 & $7.569,65$ & --- \\
1000 & $7.803,84$ & --- \\
2000 & $7.969,97$ & $7.970,00$ \\
4000 & $8.370,14$ & --- \\
6000 & $8.770,23$ & --- \\
8000 & $9.004,26$ & --- \\
10000 & $9.170,30$ & --- \\
\hline
\end{tabular}


A partir dos dados da Tabela 5, verifica-se que os valores obtidos do estudo de caso estão em concordância com dados relatados em outros estudos.

Para um tempo de retorno de 10 anos, os valores das vazões calculados foram de 5282,40 $\mathrm{m}^{3} / \mathrm{s}$ para o estudo de caso e 5280 $\mathrm{m}^{3} / \mathrm{s}$ para dados da literatura. Enquanto, para o período de recorrência de 10000 anos, os valores das vazões do estudo de caso e da literatura foram estimados como sendo $9.299,08 \mathrm{~m}^{3} / \mathrm{s}$ e $9300 \mathrm{~m}^{3} / \mathrm{s}$, respectivamente.

A Figura 2 mostra a distribuição de Gumbel, para o período de retorno x vazões estimadas do estudo de caso.

Figura 2. Distribuição de Gumbel do estudo de caso.

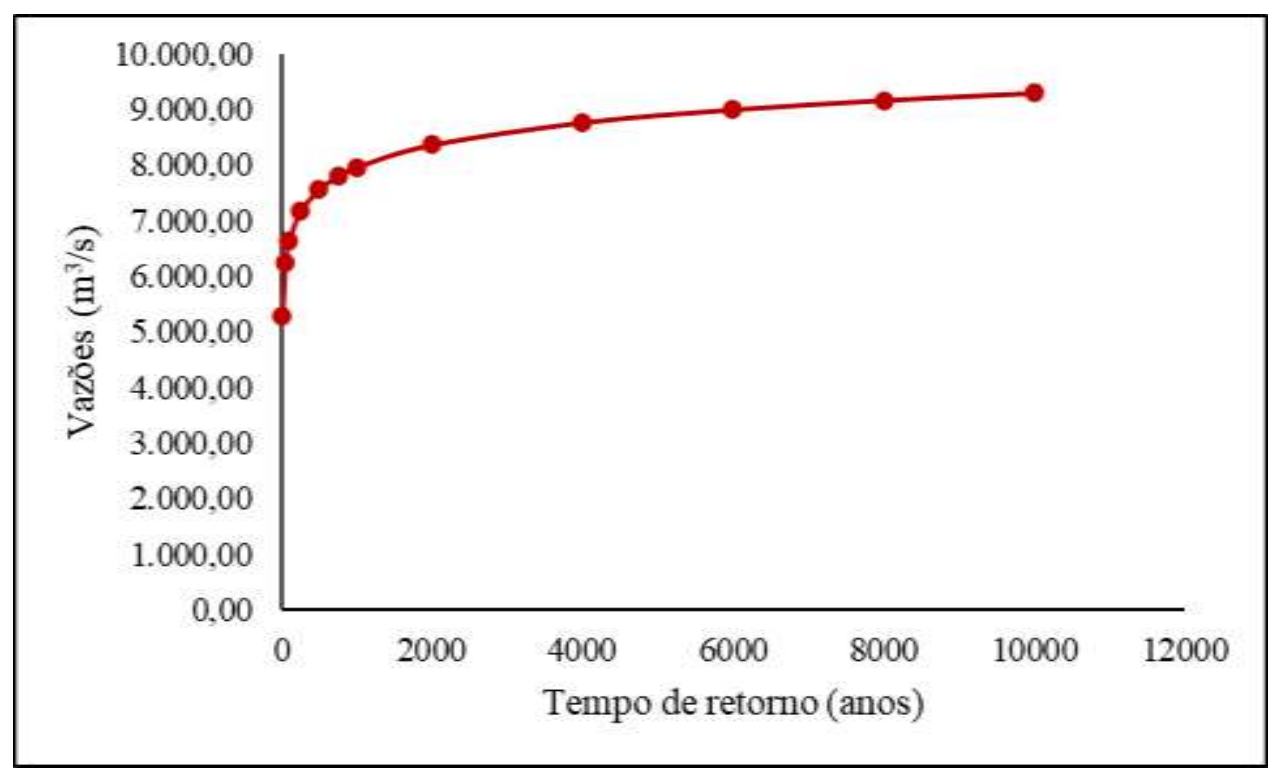

Fonte: Autores.

\section{Conclusão}

As obras de hidráulicas, principalmente as de grande porte, uma vez que envolvem parâmetros complexos, necessitam de estudos robustos antes do desenvolvimento do empreendimento. Entre os estudos realizados estão os de vazão de projeto. É necessário realizar estudos que forneçam previsões sobre a quantidade de água que alimentam o reservatório, bem como a frequência de ocorrência desse fenômeno. Análises probabilísticas podem auxiliar no dimensionamento de estruturas hidráulicas e, assim minimizar os erros de projeto e possíveis catástrofes. O método de distribuição de Gumbel é uma das ferramentas probabilísticas mais utilizadas para cálculo de eventos extremos. Neste trabalho de pesquisa, distribuição de Gumbel para valores extremos foi utilizada com êxito para analisar os dados das vazões máximas de uma série histórica de 37 anos da estação Tabajara, do rio Ji-Paraná, localizado no município de Machadinho d'Oeste - RO. Para um tempo de retorno de 10 anos, os valores das vazões calculados foram de 5282,40 $\mathrm{m}^{3} / \mathrm{s}$, enquanto para o período de recorrência de 10000 anos, os valores das vazões foram estimados como sendo 9.299,08 $\mathrm{m}^{3} / \mathrm{s}$. Os valores obtidos no estudo de caso estão em concordância com dados relatados em outros estudos. O método de Gumbel demonstra potencial para aplicações em outros sistemas que necessitem de uma abordagem probabilística como na estimativa de precipitações máximas em dada região. Por outro lado, o método de Gumbel também pode ser explorado no caso de precipitações mínimas. Assim, o trabalho de pesquisa além de possibilitar o leitor uma abordagem abrangente para caso de cheias máximas de projeto usando a distribuição de Gumbel para eventos máximos e oferece subsídios para trabalhos futuros usando outras abordagens. 


\section{Agradecimentos}

Os autores gostariam de agradecer ao Centro Universitário INTA - UNINTA e a Agência Nacional das Águas.

\section{Referências}

Affonso, V., Faria, G. A., Lopes, B. G., Tsutsumoto, N. Y., Fonseca, A. D. \& Felizardo, L. M. (2020). Análise dos dados de precipitação máxima no noroeste paulista pela teoria dos valores extremos. Research, Society and Development, 9 (10), e9709109396.

ANA. (2021). Banco de Dados Hidrológicos. Agência Nacional das Águas (ANA). http://hidroweb.ana.gov.br/.

Bhagat, N. (2017). Flood Frequency Analysis Using Gumbel's Distribution Method: A Case Study of Lower Mahi Basin, India. Journal of Water Resources and Ocean Science, 6(4), 51-54.

CBB. (2001). Comitê Brasileiro de Barragens (CBB). Guia Básico de Segurança de Barragens.

Estrela, C. (2018). Metodologia Científica: Ciência, Ensino, Pesquisa. Editora Artes Médicas.

Ganamala, K. \& Kumar, P. S. (2017). A Case Study on Flood Frequency Analysis, International Journal of Civil Engineering and Technology, 8(4), 17621767.

Gumbel, E. J. (1941). The return period of flood flows. The Annals of Mathematical Statistics, 12(2), 163-190.

Filho, D. F. F., Rodrigues, R. S. S., Silva, M. N. A., Fernandes, L. L. \& Crispim, D. L. (2020). Aplicação de diferentes métodos de determinação de curvas de intensidade-duração-frequência no município de Belterra no estado do Pará, Brasil. Research, Society and Development, 9(2), e77922073.

Franco, C. S., Marques, R. F. P. V., Oliveira, A. S. \& Oliveira, L. F. C. (2014). Distribuição de probabilidades para precipitação máxima diária na Bacia Hidrográfica do Rio Verde, Minas Gerais. Revista Brasileira de Engenharia Agrícola e Ambiental, 18(7), $735-741$.

Jesus, J. B. \& Nascimento, Y. S. (2020). Tempo de retorno e espacialização das precipitações máximas pelo método dos momentos para o estado da Bahia. Engenharia Sanitária e Ambiental, 25(1), 127-131.

Júnior, J. A. J., Mello, C. M. \& Alves, G. J. (2015). Eventos extremos de precipitação no Alto Rio Grande, MG: Análise probabilística. Revista Brasileira de Engenharia Agrícola e Ambiental, 19(4), 301-308.

Kamal, V., Mukherjee, S., Singh, P., Sen, R., Vishwakarma, C. A., Sajadi, P., Asthana, H. \& Rena, V. (2017). Flood frequency analysis of Ganga river at Haridwar and Garhmukteshwar. Applied Water Science, 7, 1979-1986.

Koutsoyiannis, D. (2004). Statistics of extremes and estimation of extreme rainfall: I. Theoretical investigation. Hydrological Sciences Journal, 49(4), 590.

Marques, R. F. P. V., Mello, C. R., Silva, A. M., Franco, C. S. \& Oliveira, A. S. (2014). Performance of the probability distribution models applied to heavy rainfall daily events. Ciência e Agrotecnologia, 38(4), 335-342.

Mercadante, O. C. (2018). Avaliação das metodologias do estudo de vazão máxima provável: Estudo de caso da bacia hidrográfica do rio Ji-Paraná/RO. Dissertação (Mestrado), Curso de Engenharia Civil, Universidade Federal do Rio de Janeiro, Rio de Janeiro.

Naghettini, M. \& Pinto, E. J. A. (2007). Hidrologia estatística. CPRM.

Neta, M. C. C., Veber, P. M., Manke, E. B., Machado, R. K., Damé, R. C. F. \& Teixeira-Gandra, C. F. A. (2019). Ajuste da distribuição log-pearson III às vazões máximas diárias anuais da bacia hidrográfica da lagoa Mirim/RS. Brazilian Journal of Development, 5(6), 6001-6007.

Nobre, M. R. P. (2020). Planilha eletrônica para cálculo de cheias máximas de projeto com a distribuição de Gumbel. Monografia, Curso de Engenharia Civil, Centro Universitário INTA - UNINTA, Sobral.

Pereira, G. S. \& Caldeira, F. V. (2018). Avaliação da distribuição de Gumbel na determinação de vazões mínimas da sub-bacia do Rio Negro. Águas Subterrâneas, 32(1), 11-16.

Soares, J. A. B., Camargo, G., Giongo, P. R., Gomes, L. F., Costa, A. R. \& Silva, P. C. (2020). Estudo hidrológico das bacias hidrográficas em Santa Helena de Goiás. Brazilian Journal of Development, 6(6), 35629 - 35647.

Ximenes, P. S. M. P., Silva, A. S. A., Ashkar, F. \& Stosic, T. (2020). Ajuste de distribuições de probabilidade à precipitação mensal no estado de Pernambuco - Brasil. Research, Society and Development, 9(11), e4869119894. 